\title{
Influencia de contaminantes ambientales en la gestación humana: causante de patologías en el adulto
}

\author{
Influence of environmental pollutants on human gestation: \\ cause of adult pathologies
}

\author{
Andrei N. Tchernitchin ${ }^{\mathrm{a}, \mathrm{b}}$, Leonardo Gaete ${ }^{\mathrm{b}}$
}

aDepartamento de Medio Ambiente, Colegio Médico de Chile

${ }^{b}$ Facultad de Medicina, Universidad de Chile

Recibido el 23 de mayo de 2018; aceptado el 29 de septiembre de 2018

\begin{abstract}
Resumen
En el presente articulo se revisan los mecanismos del imprinting epigenético mediante el cual se producen los efectos diferidos generados por la exposición prenatal o infantil temprana a agentes químicos contaminantes. Se revisaron las bases de datos Pubmed y Embase para identificar estudios publicados entre 2005 y 2018, junto a artículos considerados pioneros en este ámbito. Se incluyeron además, datos generados en nuestro Laboratorio. Como fuente de información secundaria se citan normas chilenas de concentraciones de algunos contaminantes en agua potable publicados por el Ministerio de Salud de Chile. Se describen cambios en la metilación de diversos genes causados por exposición prenatal o infantil temprana a algunos contaminantes ambientales relevantes en Chile: arsénico, plomo, ftalatos y fenoles, y se mencionan algunas de las enfermedades orgánicas y cambios neuroconductuales que se desarrollan más tarde en la vida como consecuencia de dichas exposiciones. Se sugiere que un mayor conocimiento de los factores ambientales y una mejor educación de la población, permitirían una protección más adecuada de embarazadas y lactantes, en especial durante las ventanas de susceptibilidad y que los pediatras y obstetras, serían los profesionales mejor indicados para desarrollar estas acciones. Se sugiere además la necesidad de adecuar normas ambientales y aumentar la fiscalización de contaminantes y sus fuentes, para prevenir el deterioro de la salud de las futuras generaciones.
\end{abstract}

Palabras clave: Contaminación ambiental; salud humana; imprinting; infancia; prenatal

\section{Keywords:}

Environmental pollution; human health; imprinting; childhood; prenatal 
published by the Ministry of Health of Chile are cited. Changes are described in the methylation of diverse genes caused by prenatal or early childhood exposure to some relevant environmental pollutants in Chile such as arsenic, lead, phenols, and phthalates, and some of the organic diseases and neurobehavioral changes that occur later in life as a consequence of these exposures are mentioned. We suggest that a wider knowledge of environmental factors and better education of the population would allow a more adequate protection of pregnant women and infants especially during the windows of susceptibility, and that pediatricians and obstetricians would be in the best position to develop these actions. We also suggest the need to adapt environmental standards and increase the control of pollutants and their sources to prevent health deterioration of future generations.

Generalidades sobre efectos diferidos por exposición perinatal a través del mecanismo del imprinting epigenético

La exposición prenatal o postnatal temprana a diversos agentes que presenten actividad hormonal determina cambios cualitativos y cuantitativos en receptores hormonales de diversos tipos celulares'. La exposición debe ocurrir durante las denominadas "ventanas de susceptibilidad" que ocurren en tiempos específicos para cada tipo celular y para cada receptor hormonal. Estas alteraciones, que persisten de por vida, son inducidas por el mecanismo del imprinting epigenético (o programación celular) ${ }^{2,3}$. Estudios realizados en nuestro país ${ }^{2-5}$ y por otros autores ${ }^{6}$, han demostrado que no solo los compuestos de acción hormonal, sino que además otros agentes, que no poseen actividad hormonal (plomo, arsénico, bifenilos policlorinados, ftalatos) también pueden activar el mecanismo del imprinting. Este mecanismo implica cambios en los patrones de expresión normales de numerosos genes por procesos como la metilación o desmetilación del ADN y por otros procesos moleculares. Estas alteraciones determinan, entre otros efectos, una alteración del número y/o las características de los diversos receptores hormonales o de neurotransmisores (Para revisión bibliográfica, ver 5).

Los primeros efectos mediados por el mecanismo del imprinting epigenético fueron descritos en animales experimentales por el biólogo húngaro Csaba y colaboradores $\left.{ }^{1,7-8}\right)$. En la especie humana hay relativamente pocos estudios que demuestran efectos diferidos de la exposición perinatal a agentes químicos, tanto en tejido embrionario y placenta como estudios clínicos y de laboratorio en períodos más tardíos de la vida. La primera patología demostrada en humanos por el ginecoobstetra estadounidense Herbst fue el desarrollo de adenocarcinomas cérvico-vaginales de células claras en mujeres jóvenes prenatalmente expuestas a dietilestilbestrol' ${ }^{9}$. Se ha propuesto que las alteraciones epigenéticas permanentes inducidas a través de metilaciones en cerebros de pacientes afectados por las enfermedades de Parkinson ${ }^{10}$, Alzheimer ${ }^{11}$ y Huntington ${ }^{12}$, y pro- bablemente, esclerosis múltiple ${ }^{13,14}$ explican el desarrollo de estas patologías. Para el sistema nervioso central, se ha planteado la hipótesis que factores epigenéticos pueden afectar la programación de células cerebrales y condicionar su susceptibilidad o resistencia para desarrollar diversas patologías neurodegenerativas ${ }^{15}$.

Entre los agentes inductores de imprinting están los diversos compuestos de acción hormonal, fármacos, drogas de abuso, contaminantes ambientales y algunos compuestos presentes en los alimentos. En este último caso, como ejemplo, la exposición prenatal a ingesta materna de grandes cantidades de grasas induce "memoria para el colesterol"16 y aumenta en forma importante los niveles de estrógeno en mujeres prenatalmente expuestas, lo cual es un factor de riesgo para el desarrollo de cáncer de mama ${ }^{17}$.

A continuación se describen algunos efectos adversos generados por la exposición prenatal o infantil temprana a contaminantes ambientales relevantes y no siempre reconocidos en Chile.

\section{Arsénico}

Se ha descrito que la exposición prenatal a arsénico (casi $900 \mu \mathrm{g} / \mathrm{L}$ en agua potable de Antofagasta entre 1958 y 1970) causa cambios irreversibles en el aparato respiratorio que determinan un aumento en 46 veces de la mortalidad por bronquiectasias entre los 30 y 49 años de edad, pero sólo 12 veces a los que nacieron de madres expuestas a alrededor de $100 \mu \mathrm{g} / \mathrm{L}$ (años $1950 \mathrm{a}$ 1957), aunque después del nacimiento los infantes hayan vivido expuestos a casi $900 \mu \mathrm{g} / \mathrm{L}^{18}$. Por otro lado, se ha descrito en Bangladesh una asociación entre exposición a arsénico durante la gestación y el aumento de enfermedades infecciosas durante la edad infantil, especialmente infecciones respiratorias bajas y diarrea ${ }^{19}$. La inmunosupresión, que se produce en personas prenatalmente expuestas ${ }^{20}$, sería el factor predisponente.

Recientemente, en mujeres taiwanesas parturientas $(\mathrm{n}=64)$ se demostró una correlación entre niveles de arsénico en orina y patrones de metilación de ADN en sangre de cordón umbilical. Los genes cuya expresión se ve alterada están involucrados en: vías de señalización, diabetes tipo I y II e interacción con ligandos y 
receptores neuroactivos. Particularmente encontraron 5 marcadores asociados con lipoproteínas de baja densidad (LDL), que podrían ser candidatos a marcadores para enfermedad cardiovascular y diabetes en poblaciones expuestas a arsénico ${ }^{21}$.

\section{Plomo}

Los efectos actualmente más relevantes de la exposición a plomo en las primeras etapas del desarrollo humano son los efectos sobre el sistema nervioso central. Causa una disminución del coeficiente de inteligencia (CI), dificultades en el aprendizaje y fracasos escolares $^{22,23}$. Este descenso del CI ya se observa a partir de una plumbemia de $5 \mu \mathrm{g} / \mathrm{dL}^{24}$. La exposición perinatal o infantil a plomo determina el desarrollo de una personalidad hiperactiva y agresiva ${ }^{25}$. El aumento del nivel de plomo en tibia es reflejo de exposición prenatal -dado que éste se incorpora al hueso prácticamente de por vida ya que esta pieza se osifica al nacer- se ha asociado a un aumento del riesgo de conductas antisociales y delictivas $^{26}$. Los volúmenes anuales de plomo importados en diversos países para ser adicionados a la bencina se han correlacionado con diversos índices de criminalidad en países como EEUU, Canadá, Francia, Gran Bretaña, Italia, Alemania Occidental, Finlandia, Australia y Nueva Zelandia. La correlación que mejor se ajustaba mostraba un desfase de 19 años entre los volúmenes de plomo importados y los índices delictuales, los que en los países investigados incrementaron al aumentar la importación de plomo, y disminuyeron al disminuir dicha importación ${ }^{27}$. La exposición prenatal a plomo en animales de experimentación causa durante la vida adulta un aumento de afinidad de receptores delta opiáceos cerebrales $^{28}$, lo que nos permitió proponer la hipótesis que la exposición a temprana edad a plomo podía facilitar la adicción a drogas de abuso opiáceas en países con altos niveles de contaminación con plo$\mathrm{mo}^{2}$. El incremento de la respuesta dopaminérgica y de ácido 5-indolacético a anfetamina en ratas por efecto de exposición a plomo ${ }^{29}$ nos sugirió que la exposición humana a plomo también podía facilitar la adicción a otras drogas estimulantes ${ }^{2}$. Nuestra hipótesis fue más tarde confirmada en diversos estudios experimentales de otros autores ${ }^{30-35}$.

La asociación entre bajas plumbemias en mujeres gestantes y metilación del ADN en sangre de cordón umbilical, ha permitido demostrar que la exposición prenatal a plomo, aún a muy bajos niveles, puede modificar los patrones de metilación del ADN y activar los mecanismos que explican los efectos diferidos de dicha exposición ${ }^{36}$.

\section{Ftalatos y fenoles}

Estos compuestos, por su estructura química, son considerados disruptores endocrinos, y en consecuen- cia pueden causar efectos adversos durante los últimos meses de la vida prenatal o durante la vida infantil temprana por el mecanismo del imprinting epigenético. Uno de los ftalatos de alto peso molecular, el dietilhexilftalato (DEHF), es utilizado en pisos plásticos, papel mural y dispositivos médicos ${ }^{37,38}$; un ftalato de bajo peso molecular, el dietilftalato (DEF) se usa como solvente en productos como lociones, lacas, barnices y recubrimientos ${ }^{39}$; y uno de los fenoles más ampliamente estudiados, el bisfenol A (BFA), se emplea en la manufactura de plásticos de policarbonato, papel térmico y de resinas epóxicas utilizadas en el revestimiento de latas de alimento ${ }^{40}$.

Un estudio en mujeres de Boston con antecedentes de exposición a ftalatos y fenoles durante el primer trimestre de su embarazo, demostró la correlación entre concentraciones de ftalatos y fenoles en orina con algunos biomarcadores de metilación del ADN en placenta y la expresión del gen paterno de IGF-2 y de un gen materno (H19), que codifica para un ARN responsable de la inhibición de proliferación celular ${ }^{41,42}$. Se demostró que la exposición prenatal a ftalatos y fenoles altera las tasas de metilación de estos dos genes humanos en placenta. La exposición prenatal a DEHF y DEF se asoció también con imprinting aberrante en mujeres recién nacidas, no así en varones ${ }^{6}$.

Varios estudios han examinado la asociación entre exposición a ftalato en el útero y el desarrollo del aparato reproductivo de hombres. En uno de ellos se encontró que niveles urinarios maternos de 4 metabolitos de ftalato, (monoetil ftalato, mono-n-butil ftalato, monobencil ftalato y monoisobutil ftalato) durante el tercer trimestre, se asociaron con distancia ano-genital disminuida en bebés varones ${ }^{43}$. Sin embargo, en un estudio reciente, la exposición a otro metabolito, el DEHF, sólo en el primer trimestre estaba asociada con distancia ano-genital disminuida ${ }^{44}$. A pesar de las diferencias en el periodo de exposición, el efecto observado es consistente con lo que se sabe actualmente sobre desarrollo reproductivo masculino, ya que la distancia ano-genital está determinada por acción androgénica durante la ventana de programación masculinizante, que en los humanos se estima es entre 7-15 semanas de desarrollo en el útero ${ }^{45}$.

En un estudio de corte transversal de exposición perinatal al ftalato, se informó que las concentraciones de monoetil-ftalato y mono-n-butil ftalato en leche materna se asociaron con aumento de la razón hormona luteinizante/testosterona libre y de globulina de unión a hormonas sexuales, SHBG en bebés varones a los tres meses de edad ${ }^{46}$.

En ciudad de México se hizo un seguimiento a 250 niños (8 a 14 años), nacidos de madres en las que se analizaron metabolitos de ftalato en orina materna y concentraciones de BFA durante el primer, segundo 
y tercer trimestre de embarazo. Los niveles de BFA y de varios metabolitos de ftalatos (monobenzil ftalato, mono-3-carboxiopropil ftalato y la suma de mono-nbutil ftalato y mono isobutil ftalato) durante el tercer trimestre, se asociaron con reducción de parámetros de adrenarquia (maduración testicular, aparición de vello pubiano) y con un aumento de SHBG sérica. Por el contrario, la exposición a DEHP en el primer trimestre, demostró un aumento de estradiol sérico peripuberal ${ }^{47}$. En otros dos estudios se examinó la asociación entre marcadores de exposición uterina a ftalatos y concentración de hormonas reproductivas en cordón umbilical, uno de los cuales concluyó que no había relación entre niveles de ftalato urinario materno y niveles hormonales en recién nacidos ${ }^{48}$. Sin embargo, en el otro estudio se reportó asociación entre los niveles de mono-etil-ftalato, en sangre materna del tercer trimestre con reducción de la razón testosterona/estradiol e inhibina B entre los hombres ${ }^{49}$.

El BFA es un disruptor endocrino cuya exposición prenatal en animales de experimentación determina diversos cambios irreversibles en la fisiología de la reproducción. En el ser humano, se ha descrito una asociación entre la exposición prenatal a BFA y síntomas de depresión y ansiedad en niños varones entre los 10 a 12 años de edad, pero no en niñas ${ }^{50}$. Además, la exposi- ción a BFA durante el tercer trimestre del embarazo se ha asociado a reducción de parámetros de adrenarquia (maduración testicular, aparición de vello pubiano) y con un aumento de SHBG sérica peripuberal ${ }^{47}$.

\section{Conclusiones}

Numerosas enfermedades de los adultos tienen su origen en la exposición perinatal a agentes inductores del imprinting epigenético. El mejor conocimiento de este mecanismo por profesionales del área de la medicina, especialmente del ámbito de la pediatría y de la obstetricia, permitiría orientar la educación de la población y en especial la de sus pacientes para ayudarles a prevenir la exposición durante las ventanas de susceptibilidad (prenatal e infantil temprana). Este conocimiento también debería alcanzar a los legisladores con el fin de lograr el adecuamiento de normas y estándares ambientales, y la implementación de medidas de prevención durante el período de vulnerabilidad, lo que permitiría mejorar las perspectivas de salud para las futuras generaciones.

\section{Conflicto de intereses}

Los autores declaran no tener conflicto de intereses.

\section{Referencias}

1. Csaba G, Inczefi-Gonda A, Dobozy O. Hormonal imprinting by steroids: a single neonatal treatment with diethylstilbestrol or allylestrenol gives rise to a lasting decrease in the number of rat uterine receptors. Acta Physiol Hung. 1986;67:207-12.

2. Tchernitchin AN, Tchernitchin N. Imprinting of paths of heterodifferentiation by prenatal or neonatal exposure to hormones, pharmaceuticals, pollutants and other agents or conditions. Med Sci Res. 1992;20:391-7.

3. Tchernitchin AN, Tchernitchin NN, Mena MA, Unda C, Soto J. Imprinting: perinatal exposures cause the development of diseases during the adult age. Acta Biol Hung. 1999;50:425-40.

4. Tchernitchin AN, Gaete L, Bustamante R, Báez A. Effect of prenatal exposure to lead on estrogen action in the prepubertal rat uterus. ISRN Obstet Gynecol; 2011:329692.

5. Tchernitchin AN, Gaete L, Bustamante $\mathrm{R}$, Sorokin YA. Adulthood prenatally programmed diseases - Health relevance and methods of study. En: Protein Purification and Analysis I. Methods and
Applications, Hong Kong: iConcept Press, 2013;217-58.

6. LaRocca J, Binder AM, Mc Elrath TF, Michels KB. The impact of first trimester phthalate and phenol exposure on IGF2/ H19 genomic imprinting and birth outcomes. Environ Res. 2014;133:396406.

7. Csaba G, Nagy SU. Plasticity of hormone receptors and possibility of their deformation in neonatal age. Experientia. 1976;32:651-2.

8. Csaba G, Dobozy O. The sensitivity of sugar receptor analysis in adult animals of influences exerted at neonatal age. Endokrinologie. 1977;69:227-32.

9. Herbst AL. Clear cell adenocarcinoma and the current status of DES-exposed females. Cancer. 1981;48:484-8.

10. Masliah E, Dumaop W, Galasko D, Desplats P. Distinctive patterns of DNA methylation associated with Parkinson disease. Epigenetics. 2013;8:1030-8.

11. van den Hove DL, Chouliaras L, Rutten BP. The role of 5-hydroxymethylcytosine in aging and Alzheimer's disease: current status and prospects for future studies. Curr Alzheimer Res. 2012;9:545-9.

12. Wang F, Yang Y, Lin X, et al. Genomewide loss of $5-\mathrm{hmC}$ is a novel epigenetic feature of Huntington's disease. Hum Mol
Genet. 2013;22:3641-53.

13. Beecham AH, Patsopoulos NA, Xifara DK, et al. Analysis of immune-related loci identifies 48 new susceptibility variants for multiple sclerosis. Nat Genet. 2013;45:1353-60.

14. Calabrese R, Valentini E, Ciccarone $\mathrm{F}$, et al. TET2 gene expression and 5 -hydroxymethylcytosine level in multiple sclerosis peripheral blood cells. Biochim Biophys Acta. 2014;1842: 1130-6.

15. Faa G, Marcialis MA, Ravarino A, Piras M, Pintus MC, Fanos V. Fetal programming of the human brain: is there a link with insurgence of neurodegenerative disorders in adulthood? Curr Med Chem. 2014;21:3854-76.

16. Brown SA, Rogers LK, Dunn JK, Gotto AM Jr, Patsch W Development of cholesterol homeostatic memory in the rat is influenced by maternal diets. Metabolism. 1990;39:468-73.

17. Hilakivi-Clarke L, Clarke R, Lippman ME. Perinatal factors increase breast cancer risk. Breast Cancer Res Treat. 1994;31:273-84.

18. Smith AH, Marshall G, Yuan Y, et al. Increased mortality from lung cancer and bronchiectasis in young adults after 
exposure to arsenic in utero and in early childhood. Environ Health Perspect. 2006;114:1293-6.

19. Rahman A, Vahter M, Ekström EC, Persson LÅ. Arsenic exposure in pregnancy increases the risk of lower respiratory tract infection and diarrhea during infancy in Bangladesh. Environ Health Perspect. 2011;119:719-24.

20. Ahmed S, Mahabbat-e Khoda S, Rekha RS, et al. Arsenic-associated oxidative stress, inflammation, and immune disruption in human placenta and cord blood. Environ Health Perspect. 2011;119:258-64.

21. Kaushal A, Zhang H, Karmaus WJJ, et al. Genome-wide DNA methylation at birth in relation to in utero arsenic exposure and the associated health in later life. Environ Health. 2017; 16: 50,11p.

22. Rothenberg SJ, Schnaas L, Cansino-Ortiz $S$, et al. Neurobehavioral deficits after low level lead exposure in neonates: the Mexico City pilot study. Neurotoxicol Teratol. 1989;11:85-93.

23. Needleman HL, Schell A, Bellinger D, Leviton A, Allred EN. The long-term effects of exposure to low doses of lead in childhood. An 11-year follow-up report. N Engl J Med. 1990;322:83-8.

24. Tarr H, Raymond RE, Tufts M. The Effects of Lead Exposure on school outcome among children living and attending Public Schools in Detroit, MI. https://www.edweek.org/media/ detroitlead.pdf , 2009. Accedido: 12 de mayo de 2018.

25. Tchernitchin AN, Lapin N, Molina L, et al. Human exposure to lead in Chile. Rev Environ Contam Toxicol. 2005;185:93139.

26. Needleman HL, Riess JA, Tobin MJ, Biesecker GE, Greenhouse JB. Bone lead levels and delinquent behavior. J Am Med Assn. 1996;275:363-9.

27. Nevin R. Understanding international crime trends: the legacy of preschool lead exposure Environ Res. 2007;104:315-36.

28. McDowell J, Kitchen I. Perinatal lead exposure alters the development of $\delta$-but not $\mu$-opioid receptors in rat brain. $\mathrm{Br} \mathrm{J}$ Pharmacol. 1988; 94: 933-7.

29. Lasley SM, Greenland RD, Minnema DJ, Michaelson IA. Altered central monoamine response to $\mathrm{D}$-amphetamine in rats chronically exposed to inorganic lead. Neurochem Res. 1985;10:933-44.

30. Kitchen I, Kelly M. Effect of perinatal lead treatment on morphine dependence in the adult rat. Neurotoxicology. 1993;14:125-9.

31. Rocha A, Valles R, Cardon AL, Bratton GR, Nation JR. Enhanced acquisition of cocaine self-administration in rats developmentally exposed to lead. Neuropsychopharmacology. 2005;30:2058-64.

32. Nation JR, Miller DK, Bratton GR. Developmental lead exposure alters the stimulatory properties of cocaine at PND 30 and PND 90 in the rat. Neuropsychopharmacology. 2000;23:44454.

33. Nation JR, Cardon AL, Heard HM, Valles R, Bratton GR. Perinatal lead exposure and relapse to drug-seeking behavior in the rat: a cocaine reinstatement study. Psychopharmacology. 2003; 168:236-43.

34. Nation JR, Smith KR, Bratton GR. Early developmental lead exposure increases sensitivity to cocaine in a self administration paradigm. Pharmacol Biochem Behav. 2004;77:127-35.

35. Clifford PS, Hart N, Thompson J, et al. Prenatal lead exposure enhances methamphetamine sensitization in rats. Pharmacol Biochem Behav. 2009;93:1659.

36. Wu S, Hivert MF, Cardenas A, et al. Exposure to low levels of lead in utero and umbilical cord blood DNA methylation in Project Viva: An epigenome-wide association study. Environ Health Perspect. 2017;125:087019, 10p.

37. Hauser R, Calafat AM. Phthalates and human health. Occup Med. 2005;62:80618.

38. Koch HM, Calafat AM. Human body burdens of chemicals used in plastic manufacture. Philos Trans R Soc Lond B Biol Sci. 2009;364:2063-78.

39. Meeker JD, Sathyanarayana S, Swan SH. Phthalates and other additives in plastics: human exposure and associated health outcomes. Philos. Trans. R. Soc. Lond.B:Biol.Sci. 2009;364:2097-113.

40. Calafat AM, Ye X, Wong LY, Reidy JA, Needham LL. Urinary concentrations of triclosan in the U.S. population: 20032004. Environ.Health Perspect. 2008;116:
303-7.

41. Gabory A, Ripoche MA, Yoshimizu T, Dandolo L. The H19 gene: regulation and function of a non-coding RNA. Cytogenet.Genome Res. 2006;113: 188-93.

42. Gabory A, Jammes H, Dandolo L. The H19 locus: role of an imprinted noncoding RNA in growth and development. Bioessays. 2010;32:473-80.

43. Swan SH, Main KM, Liu F, et al. Decrease in anogenital distance among male infants with prenatal phthalate exposure. Environ Health Perspect. 2005;113:1056-61.

44. Martino-Andrade AJ, Liu F, Sathyanarayana S, et al. Timing of prenatal phthalate exposure in relation to genital endpoints in male newborns. Andrology. 2016;4:585-93.

45. Welsh M, Saunders PT, Fisken M, et al. Identification in rats of a programming window for reproductive tract masculinization, disruption of which leads to hypospadias and cryptorchidism. J Clin Invest. 2008;118:1479-90.

46. Main KM, Mortensen GK, Kaleva MM, et al. Human breast milk contamination with phthalates and alterations of endogenous reproductive hormones in infants three months of age. Environ Health Perspect. 2006;114:270-6.

47. Watkins DJ, Sánchez BN, Téllez-Rojo $\mathrm{MM}$, et al. Impact of phthalate and BPA exposure during in utero windows of susceptibility on reproductive hormones and sexual maturation in peripubertal males. Environ Health. 2017;16:69.

48. Lin LC, Wang SL, Chang YC, et al. Associations between maternal phthalate exposure and cord sex hormones in human infants. Chemosphere. 2011;83:1192-9.

49. Araki A, Mitsui T, Miyashita C, et al. Association between maternal exposure to di(2-ethylhexyl) phthalate and reproductive hormone levels in fetal blood: the Hokkaido study on environment and children's health. PLoS One. 2014;9:e109039.

50. Perera F, Nolte ELR, Wang Y, et al. Bisphenol A exposure and symptoms of anxiety and depression among inner city children at 10-12 years of age. Environ Res. 2016;151:195-202. 